\title{
Amplified fragment length polymorphism (AFLP) reveals species-specific markers in the Daphnia galeata-hyalina species complex
}

\author{
Massimiliano Gili ${ }^{1,2}$, Michael T. Monaghan ${ }^{1,3}$ \& Piet Spaak $^{1, *}$ \\ ${ }^{1}$ Department of Limnology, EAWAG, Überlandstrasse 133, Postfach 611, 8600 Dübendorf, Switzerland \\ ${ }^{2}$ Present address: Via Stazione 78, 10060 None (Torino), Italy \\ ${ }^{3}$ Present address: Molecular Systematics Laboratory, Department of Entomology, The Natural History Museum, \\ Cromwell Road, London SW7 5BD, UK \\ (*Author for correspondence: Tel.: +41-1-823-5617; Fax: +41-1-823-5315; E-mail: spaak@eawag.ch)
}

Key words: hybridization, molecular marker, backcrossing, introgression, Daphnia

\begin{abstract}
Daphnia often occur in species complexes that consist of two or more co-occurring species and their hybrids. Hybrid individuals are often capable of sexual reproduction and so backcrossing with introgression occurs. To better understand hybridization and backcrossing frequency, we sought to develop PCRbased, species-specific markers in the Daphnia galeata-hyalina species complex using amplified fragment length polymorphism (AFLP). This technique produces large numbers of reproducible markers for assessing diversity across the nuclear genome and provides several advantages over mtDNA and microsatellite approaches. We examined 28 clones of D. galeata, D. hyalina, and their hybrids isolated from Lake Constance on the Swiss-German border. Using a single AFLP primer combination we found five potential species-specific markers, defined as bands that occurred in $>80 \%$ of one parental species and $<20 \%$ of the other. Two bands appeared to be co-dominant and were present (homozygous) in D. galeata, absent in D. hyalina, and heterozygous in the hybrid. We conclude AFLP could provide enough PCR-based, speciesspecific markers to identify species, hybrids, and backcrosses from even small amounts of tissue (i.e. resting eggs).
\end{abstract}

\section{Introduction}

Determining the Daphnia species composition of European lakes is not always an easy task. Sibling species typically co-occur and often hybridize (Schwenk \& Spaak, 1995). Although attempts have been made to distinguish taxa morphologically (Flößner, 1993; Gießler, 2001), it remains difficult for non-specialists to quantify species and hybrid frequencies. With the help of genetic techniques (allozymes), Wolf \& Mort (1986) and Wolf (1987) found that certain Got (renamed to $A A T$ ) alleles were species-specific and can be used to identify hybrids and parental species within the Daphnia galeata-hyalina-cucullata complex. Gießler (1997) identified $A O$ as another diagnostic locus, provid- ing the possibility to distinguish backcrosses. Further studies have found that hybridization and backcrossing are common events in natural populations (Spaak, 1996; Schwenk, 1997; Schwenk et al., 1998). All studies published to date show evidence for backcrossing within the Daphnia galeata-hyalina-cucullata species complex, but with only two species-specific markers it remains difficult to identify individual backcrosses (but see Jankowski, 2002; Spaak et al., 2004).

Schwenk (1993) found species-specific mtDNA restriction sites, and since mtDNA is inherited maternally it can be used to investigate which species served as the maternal parent of a hybrid 
individual. In a later study Schwenk (1997) found that the directionality of hybridization was nonrandom; D. cucullata was significantly more frequently the maternal species in D. galeata-cucullata hybrids. Other studies have used mtDNA sequence information to study species composition of ephippial eggs sampled from lake sediments (Duffy et al., 2000) or for phylogenetic studies (Colbourne \& Hebert, 1996; Schwenk et al., 2000). Microsatellites have been developed for a number of Daphnia species (Ender et al., 1996; Pfrender et al., 2000; Limburg \& Weider, 2002). Microsatellites are co-dominant and often highly variable; however, sequence knowledge is required for primer design and no species-specific loci have been reported yet for Daphnia.

The amplified fragment length polymorphism technique (AFLP, Vos et al., 1995) has received increasing attention for the study of hybridization. The technique produces large numbers of reproducible markers for assessing diversity across the entire nuclear genome (Fig. 1), providing several advantages over mtDNA and microsatellite approaches. No prior knowledge of the genome is

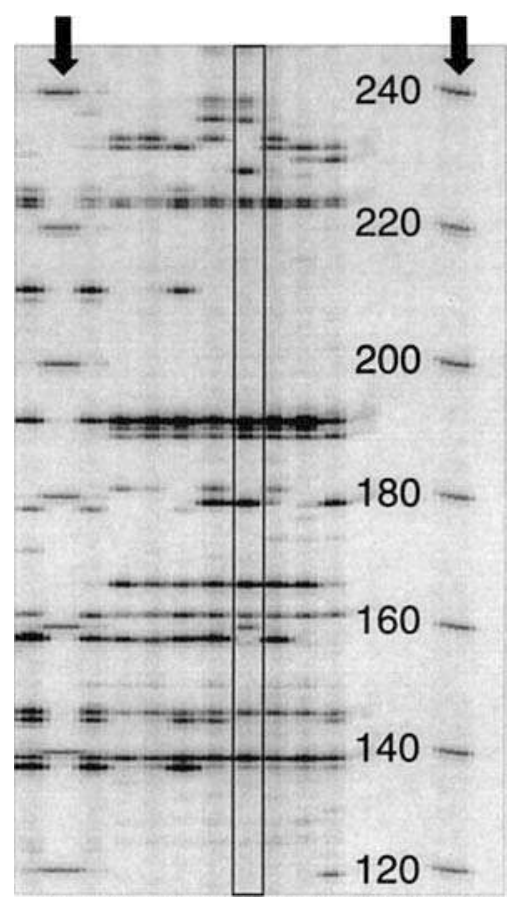

Figure 1. Part of an AFLP-gel with banding patterns of 10 individual Daphnia. One individual is enclosed with black lines. The arrows indicate the lanes in which size markers were run. The numbers indicate the size of the markers in base pairs (bp). required, thus eliminating the time required for marker development. At the intraspecific level AFLP has successfully been used to differentiate populations of animals and plants (e.g., O'Hanlon et al., 1999; Bensch et al., 2002). Most studies use AFLP only as a dominant marker (e.g., Ogden \& Thorpe, 2002); however, automated sequencers and software that measures band intensity enable the scoring of co-dominant markers, where the heterozygous locus is marked by a band with half the intensity of the homozygous locus (Castiglioni et al., 1999; Wong et al., 2001).

The goals of the present study were to investigate the utility of AFLP to quantify genetic variation in Daphnia and to identify species-specific genetic markers for D. galeata and D. hyalina. The aim was to find markers that enable the recognition of parental species, hybrids, and backcrosses. We examined 28 individuals of D. galeata, D. hyalina, and D. galeata-hyalina hybrids (based on their $A A T$ and $A O$ allozyme alleles) from Lake Constance, on the Swiss-German border, where both parental species, hybrids, and backcrosses occur together.

\section{Material and methods \\ Origin of Daphnia clones}

Daphnia clones from Lake Constance had been cultured in the laboratory for more than 1 year. We selected the clones based on the genotype for the diagnostic allozymes $A A T$ and $A O$, using clones with the $D$. galeata, D. hyalina or the hybrid genotype for both loci (Table 1). The clones represented 17 different multilocus genotypes (MLG) based on allozyme electrophoresis for the enzymes $A A T, A O, P G I$ and $P G M$.

\section{DNA extraction}

DNA was extracted using a 'salting out' procedure modified from (Sunnucks \& Hales, 1996). Daphnia were incubated with proteinase $\mathrm{K}$ in $200 \mu \mathrm{l}$ TNES buffer (50 mM Tris, $20 \mathrm{mM}$ EDTA, $400 \mathrm{mM} \mathrm{NaCl}$, $0.1 \% \mathrm{SDS}$ ) at $55^{\circ} \mathrm{C}$ for $2-3 \mathrm{~h}$. After incubation, $25 \mu \mathrm{l}$ of $5 \mathrm{M} \mathrm{NaCl}$ was added, followed by centrifugation at $3000 \mathrm{rpm}$ for $10 \mathrm{~min}$. The supernatant was transferred to new tubes and an equal volume 
Table 1. Overview of the different allozyme genotypes used in this study. All clones were included in the AFLP analysis as indicated in the last columns

\begin{tabular}{llll}
\hline Taxon & AAT-AO-PGI-PGM Genotype & No. individuals & MseI-EcoRI \\
\hline D. gal. $\times$ hyl & SF + SFMMFF & 2 & 2 \\
D. gal. $\times$ hyl. & SFSFMMFF & 1 & 1 \\
D. gal. $\times$ hyl. & SFSFMMMF & 2 & 2 \\
D. gal. $\times$ hyl. & SMSFMMFF & 1 & 1 \\
D. gal. $\times$ hyl. & SMSFMMFF+ & 2 & 2 \\
D. galeata & FFFFMFFF & 1 & 1 \\
D. galeata & FFFFMFSF & 1 & 1 \\
D. galeata & FFFFMFSM & 1 & 1 \\
D. galeata & FFFFMMFF+ & 1 & 1 \\
D. galeata & FFFFMMMF & 2 & 2 \\
D. galeata & MFFFMFSF & 1 & 1 \\
D. galeata & MMFFMFFF & 1 & 1 \\
D. galeata & MMFFMMMF & 1 & 1 \\
D. galeata & MMFFMMSF & 1 & 1 \\
D. hyalina & SSSSMMFF & 7 & 7 \\
D. hyalina & SSSSMMFF + & 1 & 1 \\
D. hyalina & SSSSMMMF+ & 2 & 2 \\
& Total & 28 & 28 \\
\hline
\end{tabular}

of chloroform was added. Following centrifugation at $8000 \mathrm{rpm}$ for $5 \mathrm{~min}$, the aqueous phase was transferred to new tubes and DNA was precipitated with an equal volume of $100 \%$ ethanol $\left(-20^{\circ} \mathrm{C}\right)$. DNA was pelleted by centrifugation (14000 rpm, $20 \mathrm{~min}$ ) and washed twice with $70 \%$ ethanol before being air-dried and resuspended in $30 \mu$ autoclaved nanopure water.

\section{AFLP analysis}

AFLP procedures, including all adapter and primer sequences, generally followed those of (Vos \& Kuiper, 1997). An aliquot of total DNA ( $8 \mu \mathrm{l})$ was digested using $20 \mathrm{U} \mathrm{MseI}$ and $10 \mathrm{U}$ EcoRI in NEB buffer No. 2 (New England Biolabs) with $1 \times$ BSA in a $20-\mu \mathrm{l}$ reaction at $37^{\circ} \mathrm{C}$ for $2-3 \mathrm{~h}$. Ligation was started immediately by adding a $5-\mu 1 \mathrm{mix}$ of $\mathrm{Mse}$ I $(27.5 \mathrm{pmol})$ and EcoRI $(2.75 \mathrm{pmol})$ adapters, T4 DNA Ligase, and $1 \times$ ligation buffer. Ligation was carried out overnight at $12^{\circ} \mathrm{C}$. Preamplification PCR was performed in $20-\mu \mathrm{l}$ volumes, with $2 \mu \mathrm{L}$ diluted restricted/ligated DNA, $1 \times \mathrm{PCR}$ buffer, $1.5 \mathrm{mM} \mathrm{MgCl}, 0.2 \mathrm{mmol}$ of each dNTP, $30 \mathrm{ng}$ $\mathrm{MseI}+\mathrm{C}$ primer and $30 \mathrm{ng}$ EcoRI. PCR was carried out with a Primus 96 Plus thermocycler
(MWG Biotech) with 2-min initial denaturation at $94^{\circ} \mathrm{C}$ followed by 35 cycles of denaturing $\left(94^{\circ} \mathrm{C}\right.$ $30 \mathrm{~s})$, annealing $\left(56^{\circ} \mathrm{C} 1 \mathrm{~min}\right)$, and extension $\left(72^{\circ} \mathrm{C}\right.$ $1 \mathrm{~min}$ ). For selective amplification, preliminary trials using several different primer combinations gave best results using MseI + CAG and Eco$\mathrm{RI}+\mathrm{AC}$ primers. Selective amplification PCR was performed in $20-\mu \mathrm{l}$ volumes, with $1 \mu \mathrm{L}$ preamplification product as template, $1 \times \mathrm{PCR}$ buffer with $1.5 \mathrm{mM} \mathrm{MgCl} 2,0.2 \mathrm{mmol}$ of each dNTP, $30 \mathrm{ng}$ MseI primer and $10 \mathrm{ng}$ EcoRI primer. EcoRI primers were labelled ( $3^{\prime}$ end) with IRD700 (MWG Biotech) infrared dye. PCR consisted of 2 min denaturation $\left(94^{\circ} \mathrm{C}\right), 13$ cycles of $30 \mathrm{~s} 94^{\circ} \mathrm{C}$, $30 \mathrm{~s} 65^{\circ} \mathrm{C}$, and $1 \mathrm{~min} 72^{\circ} \mathrm{C}$, with the annealing temperature decreasing from $65^{\circ} \mathrm{C}$ by $0.7^{\circ} \mathrm{C}$ increments in cycles $2-13$. This was followed by 26 cycles of $30 \mathrm{~s} 94^{\circ} \mathrm{C}, 30 \mathrm{~s} 56^{\circ} \mathrm{C}$, and $1 \mathrm{~min} 72^{\circ} \mathrm{C}$.

PCR products from the selective amplification were denatured with microSTOP loading buffer (Microzone Limited, East Sussex, UK). Electrophoresis and band/fragment detection was carried out on a Li-Cor 4200 LongReadIR automatic sequencer. Electrophoresis was run at $45 \mathrm{~W}$ $(\sim 1500 \mathrm{~V}), 40 \mathrm{~mA}$, and $45^{\circ} \mathrm{C}$, using $6 \%$ polyacrylamide gels and $41-\mathrm{cm}$ plates. Fragment sizes (bp) 
were determined using microSTEP 24a (Microzone Limited) fluorescent marker and Gene ImagIR v 4.03 (Scanalytics) software.

\section{Data analysis}

The AFLP bands we scored were dark, consistent and repeatable in individuals across PCR reactions and gels. We scored 75 bands (AFLP restriction sites), ranging from 70 to $307 \mathrm{bp}$ in length. Of the 75 bands, 71 were polymorphic and 12 bands occurred in only one individual. Band presence/absence was scored in a binary data matrix and a dendrogram was constructed with the complete linkage method using Statistica (StatSoft, 2001) using the dissimilarity between the clones. A similarity matrix, constructed using the Jaccard coefficient (Sneath \& Sokal, 1973), was used for a multidimensional scaling analysis (MDS) using Statistica.

Potential species-specific bands should be indicated by a high frequency in one parental species and the hybrid and a low frequency in the other parental species. Ideally bands would occur in $100 \%$ and $0 \%$ frequencies, but because (1) backcrossing occurs in this species complex, and (2) the clones we examined naturally co-occur with hybrids and parentals, we cannot exclude the occurrence of introgression in our laboratory-cultured clones. Therefore we used a criterion of $20 \%$. If a band had a frequency above $80 \%$ in both the hybrid and a parental species and a frequency below $20 \%$ in the other parental species it was considered as a potential species-specific marker.

\section{Results}

\section{Species-specific markers}

Two bands (104, $210 \mathrm{bp})$ were present in all $D$. hyalina and occurred in only $10 \%$ (104 bp) or were absent (210 bp) from D. galeata clones (Table 2). Both of these bands occurred in all hybrids (Table 2), making them strong candidates for speciesspecific markers in D. hyalina. Three bands (166, $189,190 \mathrm{bp}$ ) occurred in $\geq 80 \%$ of $D$. galeata and $\leq 20 \%$ of $D$. hyalina (Table 2 ), but all three bands were present in 62.5 or $75 \%$ of hybrids. This was lower than our $80 \%$ criterion set a priori for species-specific markers, but suggests the presence of species-specific bands masked by backcrossing and introgression that could be distinguished with additional sampling.

\section{Genetic variation}

The UPGMA dendrogram revealed two primary clusters, one with all but a single $D$. galeata clone (as defined a priori by their allozyme pattern for $A A T$ and $A O$ ) and one with $D$. hyalina and the hybrids. This second cluster was further divided into a $D$. hyalina cluster with three hybrid clones and a hybrid cluster with 1 D. galeata clone.

The two-dimensional plot generated from multidimensional scaling of the Jaccard index gave slightly more pronounced separation of parentals and hybrids (Fig. 3). D. galeata clones clustered together and were distinctly separated from the cluster of D. hyalina. The two-dimensional area of the clusters indicated genetic variation was similar for both species. Five of the hybrid clones clustered intermediate of the parental species and two hybrids clustered within the D. hyalina cluster. A single individual (X08) did not cluster with any others.

\section{Co-dominance of AFLP bands}

Two of the potential D. galeata-specific bands (166, $189 \mathrm{bp}$ ) appeared co-dominant, with nearly all hybrids exhibiting less intensity (i.e., fainter bands; Fig. 4) than the parental. The 166-bp band (see Fig. 4) was present but feint in one D. galeata and in one D. hyalina individual, suggesting they could be backcrosses (see Discussion). The 189-bp fragment was present but feint in two $D$. galeata and one D. hyalina, also suggesting the presence of backcrosses. Similar patterns were observed for the two D. hyalina-specific bands (104, $210 \mathrm{bp})$, with hybrids exhibiting reduced band intensity.

\section{Discussion}

Distinguishing among parental species and hybrids

Our results show that with AFLP analysis a clustering of taxa within the D. galeata-hyalina species complex can be achieved that is comparable to that achieved with allozymes. The Daphnia clones 
Table 2. Frequency of the 20 most frequently occurring bands, sorted by each of the three taxa examined

\begin{tabular}{|c|c|c|c|c|c|c|c|c|c|c|c|}
\hline \multicolumn{4}{|c|}{ D. hyalina sorted } & \multicolumn{4}{|c|}{ Hybrid sorted } & \multicolumn{4}{|c|}{ D. galeata sorted } \\
\hline \multicolumn{4}{|c|}{$n=10$} & \multicolumn{4}{|l|}{$n=8$} & \multicolumn{4}{|c|}{$n=10$} \\
\hline MW & D. galeata & D. gal. $\times h y l$. & D. hyalina & MW & D. galeata & D. gal. $\times h y l$. & D. hyalina & MW & D. galeata & D. gal. $\times h y l$. & D. hyalina \\
\hline 77 & 1.00 & 1.00 & 1.00 & 77 & 1.00 & 1.00 & 1.00 & 77 & 1.00 & 1.00 & 1.00 \\
\hline 139 & 1.00 & 1.00 & 1.00 & 139 & 1.00 & 1.00 & 1.00 & 113 & 1.00 & 0.75 & 1.00 \\
\hline 146 & 1.00 & 1.00 & 1.00 & 146 & 1.00 & 1.00 & 1.00 & 139 & 1.00 & 1.00 & 1.00 \\
\hline 223 & 1.00 & 1.00 & 1.00 & 191 & 1.00 & 1.00 & 0.90 & 146 & 1.00 & 1.00 & 1.00 \\
\hline 91 & 0.90 & 1.00 & 1.00 & 223 & 1.00 & 1.00 & 1.00 & 162 & 1.00 & 0.88 & 0.80 \\
\hline 210 & 0.10 & 1.00 & 1.00 & 70 & 0.90 & 1.00 & 0.90 & 166 & 1.00 & 0.63 & 0.20 \\
\hline 104 & 0.00 & 1.00 & 1.00 & 91 & 0.90 & 1.00 & 1.00 & 191 & 1.00 & 1.00 & 0.90 \\
\hline 224 & 1.00 & 0.88 & 1.00 & 137 & 0.90 & 1.00 & 0.80 & 223 & 1.00 & 1.00 & 1.00 \\
\hline 95 & 0.80 & 0.88 & 1.00 & 210 & 0.10 & 1.00 & 1.00 & 224 & 1.00 & 0.88 & 1.00 \\
\hline 158 & 0.70 & 0.88 & 1.00 & 104 & 0.00 & 1.00 & 1.00 & 70 & 0.90 & 1.00 & 0.90 \\
\hline 113 & 1.00 & 0.75 & 1.00 & 162 & 1.00 & 0.88 & 0.80 & 91 & 0.90 & 1.00 & 1.00 \\
\hline 114 & 0.90 & 0.75 & 1.00 & 224 & 1.00 & 0.88 & 1.00 & 114 & 0.90 & 0.75 & 1.00 \\
\hline 225 & 0.10 & 0.63 & 1.00 & 90 & 0.80 & 0.88 & 0.70 & 137 & 0.90 & 1.00 & 0.80 \\
\hline 191 & 1.00 & 1.00 & 0.90 & 95 & 0.80 & 0.88 & 1.00 & 189 & 0.90 & 0.63 & 0.10 \\
\hline 70 & 0.90 & 1.00 & 0.90 & 158 & 0.70 & 0.88 & 1.00 & 190 & 0.90 & 0.75 & 0.10 \\
\hline 252 & 0.00 & 0.75 & 0.90 & 145 & 0.60 & 0.88 & 0.70 & 90 & 0.80 & 0.88 & 0.70 \\
\hline 137 & 0.90 & 1.00 & 0.80 & 177 & 0.30 & 0.88 & 0.60 & 95 & 0.80 & 0.88 & 1.00 \\
\hline 162 & 1.00 & 0.88 & 0.80 & 113 & 1.00 & 0.75 & 1.00 & 158 & 0.70 & 0.88 & 1.00 \\
\hline 106 & 0.10 & 0.63 & 0.80 & 114 & 0.90 & 0.75 & 1.00 & 232 & 0.70 & 0.63 & 0.50 \\
\hline 307 & 0.00 & 0.38 & 0.80 & 190 & 0.90 & 0.75 & 0.10 & 285 & 0.70 & 0.00 & 0.00 \\
\hline
\end{tabular}

Bold bands indicate possible species-specific alleles based on our $20 \%$ criterion, where bands with a frequency above $80 \%$ in both the hybrid and a parental species and a frequency below 20 in the other parental species are defined as a potential species specific marker. MW (molecular weight) indicates fragment size.

that we classified as $D$. hyalina and $D$. galeata using the $A A T$ and $A O$ loci clustered in different groups (Figs. 2 and 3). Both UPGMA and multidimensional scaling results strongly suggest AFLP can be used to differentiate taxa in Daphnia species complexes as has been demonstrated in vertebrates (e.g., Bensch et al., 2002) and plants (e.g., Beismann et al., 1997). The fact that only one $D$. galeata clone occurred outside the $D$. galeata grouping (UPGMA, Fig. 2) and all D. galeata clustered together with multidimensional scaling (Fig. 3) suggests that our a priori designation of taxon, using two species-specific allozyme markers, probably excluded most of the backcrosses that are likely present (Spaak, 1996; Gießler et al., 1999).

One of the candidate species-specific bands (104 bp) occurred in all parental D. hyalina, all hybrids, and in no D. galeata. While this is the ideal case and therefore the best candidate for being species-specific, data from more individuals and more lakes are required for confirmation. The other four potential species-specific bands all occurred in at least some of the other parental species. This requires additional study, largely because of the occurrence of backcrossing in Lake Constance (Jankowski, 2002). When F1 or F2 hybrids backcross with a parental, the number of species-specific markers needed for correct identification increases to 5 or more markers (Boecklen $\&$ Howard, 1997). However, the results from only a single AFLP primer pair (MseI + CAG/EcoR$\mathrm{I}+\mathrm{AC})$ are encouraging, having produced five potentially species-specific bands. We also consider that our criterion for a species-specific band ( $>80 \%$ in one species, $<20 \%$ in the other) is somewhat conservative. For example, Bensch et al. (2002) used the criterion that 'markers should be at 


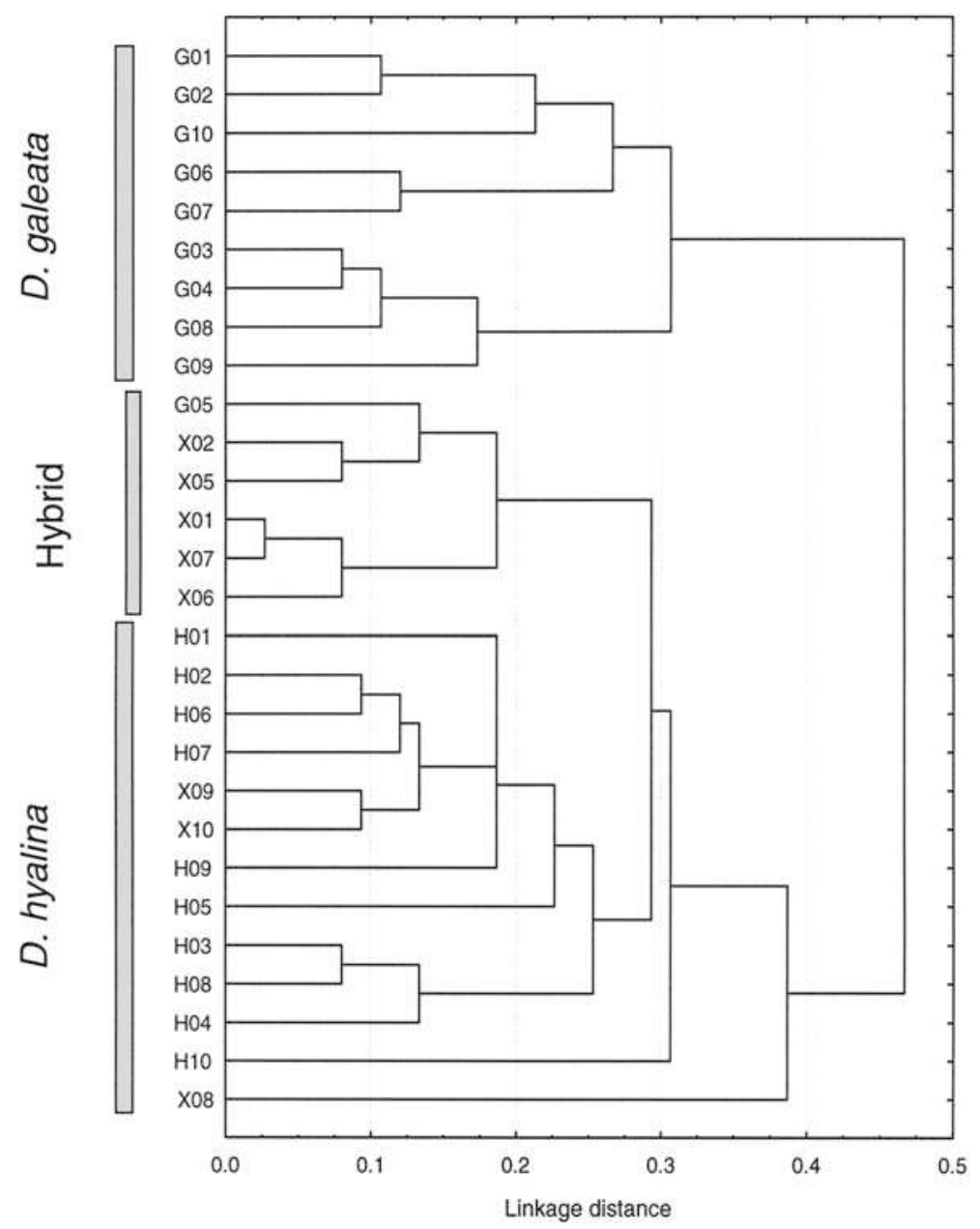

Figure 2. Dendrogram of AFLP band-sharing dissimilarity for the 28 Daphnia clones. The first letter of the clone name indicates the taxon $(\mathrm{G}=$ D. galeata $; \mathrm{H}=$ D. hyalina $; \mathrm{X}=$ interspecific hybrid $)$.

least three times as common in one of the taxa than in the other' and be present in at least $23.3 \%$ of individuals in the taxon with the highest frequency of that marker (Bensch et al., 2002).

Our results make clear that the search for species-specific markers ideally would use clones from populations where the parental species do not cooccur. The use of $D$. hyalina clones from Lake Constance was, in our opinion, justified since Lake Constance is the type location for $D$. hyalina (Leydig, 1860).

\section{Co-dominance}

Several recent studies (Castiglioni et al., 1999; Piepho \& Koch, 2000; Jansen et al., 2001; Wong et al., 2001) have reported co-dominance of AFLP restriction sites. Individuals heterozygous for an AFLP locus exhibit bands that have half the intensity of homozygous bands. Applying this to Daphnia hybrids, we found that hybrids exhibited 166 and $189 \mathrm{bp}$ bands at lower intensities than parental D. galeata (Fig. 4). This suggests that the parental has two copies and the hybrid only one copy of the restriction site as has been shown previously (e.g., Piepho \& Koch, 2000; Wong et al., 2001). Since low amounts of template DNA and sub-optimal PCR conditions also affect band intensity, standardized DNA template volumes and PCR conditions are necessary steps in using species-specific co-dominant bands to identify hybrids. Another important consideration for 


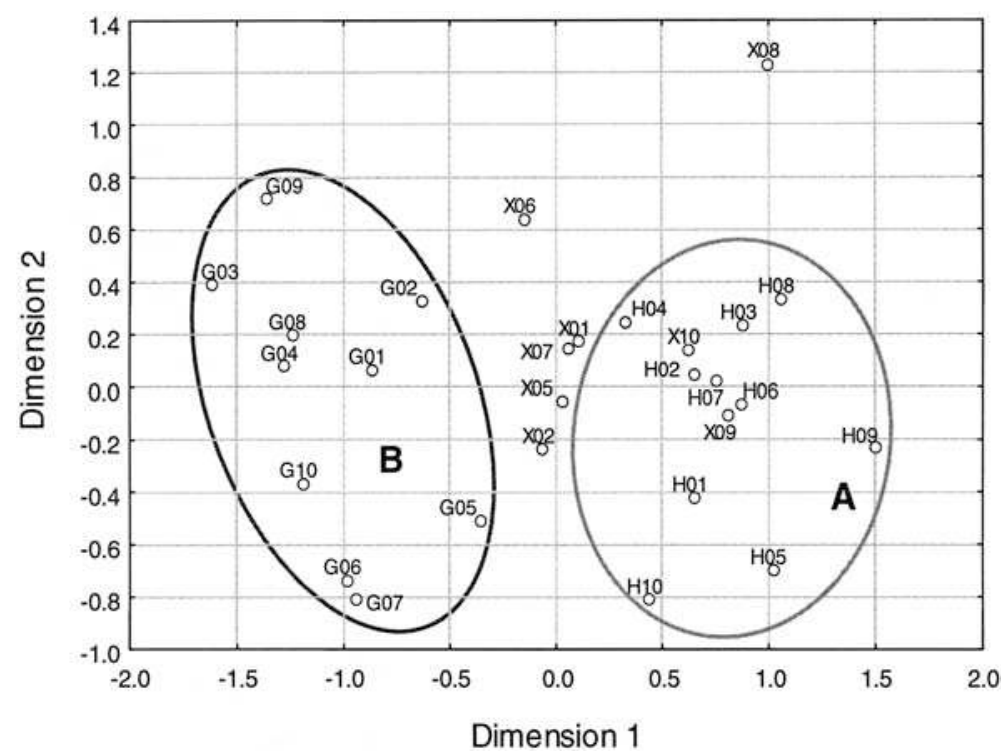

Figure 3. Two-dimensional multidimensional scaling plot of Jaccard distances calculated using the AFLP presence/absence dataset. The clones from both parental species (as defined by allozyme electrophoresis) cluster together: group A D. hyalina; group B contains the $D$. galeata clones. Most hybrid clones appear intermediate to the clusters, although 2 occur within the D. hyalina cluster.

co-dominance in AFLP is that a band with relatively low intensity for a homozygote might not be detectable for a heterozygous individual. It is therefore more likely that species-specific bands are not detected in hybrids compared to the parental species.

In summary, our data are limited, but the fact that hybrids clustered within the D. hyalina cluster and not within the $D$. galeata cluster suggests that hybrids backcross more frequently with $D$. hyalina than with $D$. galeata, as has been observed in previous studies (Jankowski, 2002). AFLP hold much promise for the delineation of hybrid-species complexes, especially for small, soft-bodies invertebrates for which the small tissue requirements of PCR techniques are well-suited. This is particularly true for Daphnia, which often are too small to examine for 5 or more allozyme loci that would be required to determine F1 and F2 backcross frequencies.

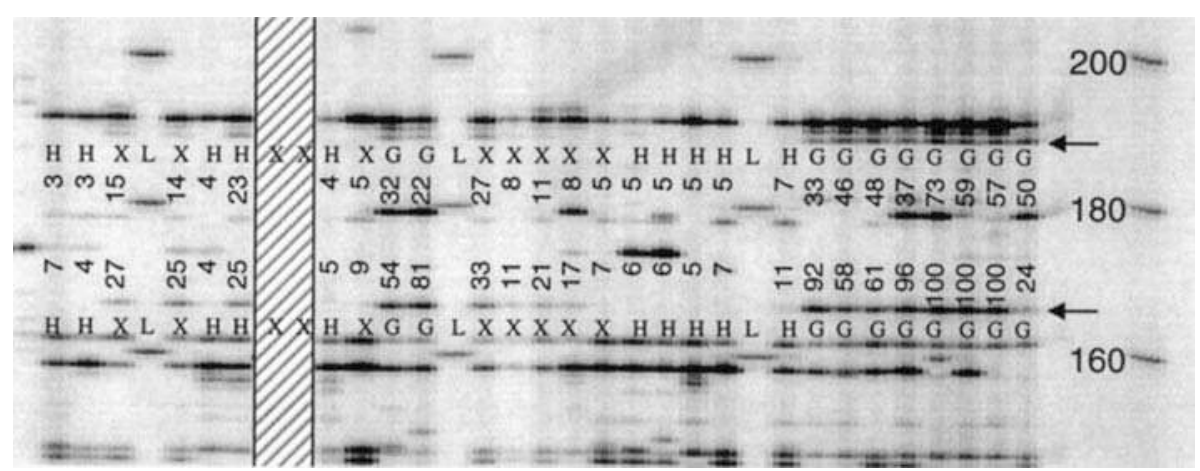

Figure 4. Fragment of AFLP gel showing the banding pattern of 28 Daphnia clones from Lake Constance. The lanes marked with L are the size marker ladder with the 160, 180 and $200 \mathrm{bp}$ markers. The bands of interest are marked with two arrows. The 166 and $189 \mathrm{bp}$ bands show a high intensity for the D. galeata $(\mathrm{G})$ clones, a low intensity for most hybrid clones $(\mathrm{X})$ and are absent for almost all the $D$. hyalina $(\mathrm{H})$ clones. The numbers in each lane indicate the intensity of the bands on a 0-100 scale. The two shaded lanes are excluded from the analysis because almost no bands were detected. 


\section{Acknowledgements}

We thank Christine Dambone for maintaining the cultures and electrophoresis analysis. Christoph Werlen, Kirsten Lawlor, Vladimir Sentchilo, Jan Roelof van der Meer, and Rik Eggen in the MIX molecular microbiology group at EAWAG provided help and support with sequencing gels. This work was supported by the Swiss Science Foundation (grants 31-50444 and 9731-65003) and by the German Science Foundation (grant RO 1008/9-1).

\section{References}

Beismann, H., J. H. A. Barker, A. Karp \& T. Speck, 1997. AFLP analysis sheds light on distribution of two Salix species and their hybrid along a natural gradient. Molecular Ecology 6: 989-993.

Bensch, S., A. J. Helbig, M. Salomon \& I. Siebold, 2002. Amplified fragment length polymorphism analysis identifies hybrids between two subspecies of warblers. Molecular Ecology 11: 473-481.

Boecklen, W. J. \& D. J. Howard, 1997. Genetic analysis of hybrid zones: numbers of markers and power of resolution. Ecology 78: 2611-2616.

Castiglioni, P., P. Ajmone-Marsan, R. van Wijk \& M. Motto, 1999. AFLP markers in a molecular linkage map of maize: codominant scoring and linkage group distribution. Theoretical and Applied Genetics 99: 425-431.

Colbourne, J. K. \& P. D. N. Hebert, 1996. The systematics of North-American Daphnia (crustacea, anomopoda) - a molecular phylogenetic approach. Philosophical Transactions of the Royal Society London Series B-Biological Sciences 351: 349-360.

Duffy, M. A., L. J. Perry, C. M. Kearns, L. J. Weider \& N. G. Hairston, 2000. Paleogenetic evidence for a past invasion of Onondaga Lake, New York, by exotic Daphnia curvirostris using mtDNA from dormant eggs. Limnology and Oceanography 45: 1409-1414.

Ender, A., K. Schwenk, T. Stadler, B. Streit \& B. Schierwater, 1996. Rapd identification of microsatellites in Daphnia. Molecular Ecology 5: 437-441.

Flößner, D., 1993. Zur Kenntnis einiger Daphnia-Hybriden. Limnologica 23: 71-79.

Gießler, S., 1997. Analysis of reticulate relationships within the Daphnia longispina species complex. Allozyme phenotype and morphology. Journal of Evolutionary Biology 10: 87105.

Gießler, S., 2001. Morphological differentiation within the Daphnia longispina group. Hydrobiologia 442: 55-66.

Gießler, S., E. Mader \& K. Schwenk, 1999. Morphological evolution and genetic differentiation in Daphnia species complexes. Journal of Evolutionary Biology 12: 710-723.

Jankowski, T., 2002. From diapause to sexual reproduction: evolutionary ecology of the Daphnia hybrid complex from
Lake Constance. Ph.D. Thesis, Universität Konstanz, Konstanz, 106 pp.

Jansen, R. C., H. Geerlings, A. J. Van Oeveren \& R. C. Van Schaik, 2001. A comment on codominant scoring of AFLP markers. Genetics 158: 925-926.

Leydig, F., 1860. Naturgeschichte der Daphniden $<$ Crustacea cladocera $>$. Laupp, Tübingen, $252 \mathrm{pp}$.

Limburg, P. A. \& L. J. Weider, 2002. 'Ancient' DNA in the resting egg bank of a microcrustacean can serve as a palaeolimnological database. Proceedings of the Royal Society London Series B-Biological Science 269: 281-287.

Ogden, R. \& R. S. Thorpe, 2002. The usefulness of amplified fragment length polymorphism markers for taxon discrimination across graduated fine evolutionary levels in Caribbean Anolis lizards. Molecular Ecology 11: 437445.

O’Hanlon, P. C., R. Peakall \& D. T. Briese, 1999. Amplified fragment length polymorphism (AFLP) reveals introgression in weedy Onopordum thistles: hybridization and invasion. Molecular Ecology 8: 1239-1246.

Pfrender, M. E., K. Spitze \& N. Lehman, 2000. Multi-locus genetic evidence for rapid ecologically based speciation in Daphnia. Molecular Ecology 9: 1717-1735.

Piepho, H. P. \& G. Koch, 2000. Codominant analysis of banding data from a dominant marker system by normal mixtures. Genetics 155: 1459-1468.

Schwenk, K., 1993. Interspecific hybridization in Daphnia: distinction and origin of hybrid matrilines. Molecular Biology and Evolution 10: 1289-1302.

Schwenk, K., 1997. Evolutionary genetics of Daphnia species complexes - hybridism in syntopy. Ph.D. Thesis, University of Utrecht, Utrecht, 141 pp.

Schwenk, K., D. Posada \& P. D. N. Hebert, 2000. Molecular systematics of European Hyalodaphnia: the role of contemporary hybridization in ancient species. Proceedings of the Royal Society London Series B-Biological Science 267: 1833-1842.

Schwenk, K., A. Sand, M. Boersma, M. Brehm, E. Mader, D. Offerhaus \& P. Spaak, 1998. Genetic markers, genealogies and biogeographic patterns in the cladocera. Aquatic Ecology 32: 37-51.

Schwenk, K. \& P. Spaak, 1995. Evolutionary and ecological consequences of interspecific hybridization in cladocerans. Experientia 51: 465-481.

Sneath, P. H. A. \& R. R. Sokal, 1973. Numerical taxonomy the principles and practice of numerical classification. Freeman, San Francisco, XV, 573 pp.

Spaak, P., 1996. Temporal changes in the genetic structure of the Daphnia species complex in Tjeukemeer, with evidence for backcrossing. Heredity 76: 539-548.

Spaak, P., A. Denk, M. Boersma \& L. J. Weider, 2004. Spatial and temporal patterns of sexual reproduction in a hybrid Daphnia species complex. Journal of Plankton Research, in press.

StatSoft, I., 2001. STATISTICA (data analysis software system), version 6. www.statsoft.com.

Sunnucks, P. \& D. F. Hales, 1996. Numerous transposed sequences of mitochondrial cytochrome oxidase I-II in aphids 
of the genus Sitobion (Hemiptera: Aphididae). Molecular Biology and Evolution 13: 510-524.

Vos, P., R. Hogers, M. Bleeker, M. Reijans, T. Van De Lee, M. Hornes, A. Frijters, J. Pot, J. Peleman, M. Kuiper \& M Zabeau, 1995. AFLP: a new technique for DNA fingerprinting. Nucleic Acids Research 23: 4407-4414.

Vos, P. \& M. Kuiper, 1997. AFLP Analysis. In CaetanoAnnoles, G. \& P. M. Gresshoff (eds), DNA Markers: Protocols, Applications, and Overviews, Wiley-VCH, New York: 115-131.
Wolf, H. G., 1987. Interspecific hybridization between Daphnia hyalina, D. galeata and D. cucullata and seasonal abundance of these species and their hybrids. Hydrobiologia 145: 213-217.

Wolf, H. G. \& M. A. Mort, 1986. Interspecific hybridization underlies phenotypic variability in Daphnia populations. Oecologia 68: 507-511.

Wong, A., M. R. Forbes \& M. L. Smith, 2001. Characterization of AFLP markers in damselflies: prevalence of codominant markers and implications for population genetic applications. Genome 44: 677-684. 